We try to publish authors' responses in the same edition with readers' comments. Time constraints might prevent this in some cases. The problem is compounded in a bimonthly journal where continuity of comment and redress are difficult to achieve. When the redress appears 2 months after the comment, 4 months will have passed since the article was published. Therefore, we would suggest to our readers that their correspondence about published papers be submitted as soon as possible after the article appears.

\section{Economics of Treatment Guidelines for Hypertension}

To the Editor: It is with great interest that we read the article by Ramsey et al, ${ }^{1}$ which reports on economic evaluation of the Sixth foint National Committee Report on the Prevention, Detection, Evaluation, and Treatment of High Blood Pressure (7NC VI). ${ }^{2}$ The authors recommend that in the future such guidelines should include economic evidence from clinical studies and other models, along with drug efficacy and safety data, in making their recommendations. The goal of JNC VI was to provide the primary care clinician with contemporary approaches to control high blood pressure using evidence-based medicine and consensus when the evidence was wanting. The evidence for the recommendations was cited and classified according to its scientific nature, and succinct considerations of economic issues and managing hypertension within managed care are presented. It is also notable that the World Health Organization-International Society of Hypertension guidelines for hypertension $^{3}$ were published in February 1999 and complement the JNC VI in many ways.

In JNC VI, we discussed the importance of evidence but recognized that the best clinical trial data have limitations. For example, trials are short and benefits accrue during a lifetime, drop-ins and drop-outs color true treatment effects, some patients must be excluded from trials, and the average blood pressure reduction in trials is modest and underestimates the benefit in those who have a greater response. We recognized the full cost of therapy beyond the cost of medication. It is obvious, however, that without a detailed economic analysis and universal agreement regarding assumptions to be entered in any economic model, for patients with uncomplicated hypertension (beyond a modest expenditure to establish a cardiovascular risk status and a few baseline tests), the cost of treatment is basically the cost of the medication. With well-controlled hypertension, patients, especially those who monitor their own blood pressure, need only one to two office visits a year and often can be cared for by a lower cost health care provider. About one half of all patients respond well to their first drug.

The cost of medication, in turn, is a function of many factors beyond the wholesale price list often used in comparisons. Currently, marketing factors, such as the nature and size of the practice(s) and the batching of drugs, affect price, and these factors change frequently. For now it is obvious that generically available diuretics, $\beta$-blockers, and angiotensin-converting enzyme inhibitors have proved their advantages. Other classes of agents must prove themselves, ie, have the same efficacy and the same or fewer side effects, show a reduction in hard end points (morbidity and mortality), and be offered at the same price or cheaper. If these agents are shown to be better at reducing morbidity and mortality, then the cost issues are of less importance. Ramsey et al do discuss the difficulties in calculating the cost of drugs in the current environment. We want to stress, however, that the treatment recommendations in JNC VI are based on the randomized clinical trials, not on cost.

We should also add that the cost of producing JNC VI was born by the National Heart, Lung, and Blood Institute (NHLBI), and only the travel expenses of the Executive Committee were reimbursed. There were more than 100 contributors and reviewers who submitted comments on the penultimate draft, and 45 professional and government agencies (the Coordinating Committee) unanimously approved the report.

One could take issue with other premises in the paper, such as the choice of baseline laboratory work, frequency of visits and laboratory tests, the importance of lifestyle changes, and choosing the drugs in 'TOMHS. ${ }^{4}$ In the meantime, we wait with anticipation for the results of ALLHAT, ${ }^{5}$ an NHLBI clinical trial that is measuring the efficacy of four classes of blood-pressure-lowering drugs in 42,500 high-risk patients. ALLHAT will respond to many of the issues raised by Ramsey et al and by JNC VI.

Sheldon Sheps, MD

Mayo Clinic, Mayo Medical School Mayo Foundation

Chair, JNC VI

Claude Lenfant, MD

Director, National Heart, Lung, and Blood Institute Chair, National High Blood Pressure Education Program Coordinating Committee Bethesda, Md

\section{References}

1. Ramsey SD, Neil N, Sullivan SD, Perfetto E. An economic evaluation of the JNC hypertension guidelines using data from a randomized controlled trial. J Am Board Fam Pract 1999; $12: 105-14$

2. The sixth report of the Joint National Committee on prevention, detection, evaluation, and treatment of high blood pressure. Arch Intern Med 1997;157:2413-46.

3. 1993 guidelines for the management of mild hypertension: memorandum from a World Health Organization/International Society of Hypertension meeting. Guidelines SubCommittee. J Hypertens 1993;11:905-18.

4. Neaton JD, Grimm RH Jr, Prineas RJ, et al. Treatment of 
mild hypertension study. Final results. Treatment of Mild Hypertension Study Research Group. JAMA 1993;270:71324.

5. Davis BR, Cutler JA, Gordon DJ, et al. Rationale and design for the Antihypertensive and Lipid Lowering Treatment to Prevent Heart Attack Trial (ALLHAT). ALLHAT Research Group. Am J Hypertens 1996;9(4 Pt 1):342-60.

The above letter was referred to the authors of the article in question, who offer the following reply.

To the Editor: Drs. Sheps and Lenfant raise several important issues in their discussion of our article. In responding, we hope to further clarify our position that economic evaluations are an important and underappreciated component of the process of creating national guidelines such as JNC VI.

As they mention, JNC VI was designed to guide primary care clinicians in the diagnosis and management of hypertension, using high-quality evidence when available and expert consensus when necessary. The force of JNC VI, however, goes well beyond the primary care physician. Adapting, implementing, and monitoring compliance with guidelines is a system-wide effort undertaken by health delivery systems and managed care groups that often have a direct economic stake in following (or not following) the guidelines. It is true that cost should be a secondary consideration for physicians when they are caring for their patients. Nevertheless, institutional decision makers cannot afford to ignore the economic considerations of their policies regarding treatment options that are laid out in guidelines.

Drs. Sheps and Lenfant state that the economic attractiveness of generic diuretics and $\beta$-blockers are selfapparent, yet a recent study shows that prescription patterns are following a trend sharply in favor of newer agents that are far more expensive than those recommended by JNC VI. ${ }^{1,2}$ Why is this so? We believe it is in large part due to the pharmaceutical industry, which suggests in its advertising that the newer agents offer substantive clinical advantages (eg, shorter time to control, fewer side effects) compared with older agents. Because physicians do apparently ignore cost in their care decisions, these new expensive agents are adopted with little regard to the cost consequences for insurers or society.

Our study was designed to show physicians and decision makers in health care delivery systems that even accounting for the nuances of hypertension care (compliance, monitoring costs), the price of the agent drives the cost of care, even in the short run. Like clinical trials, economic models have limitations in their methods and generalizability. Our model followed the recommendations of JNC VI and used data cited from this report wherever possible because we believe this report is the most internally and externally valid summary of hypertension care that is available. Of course, local costs and practice patterns will vary, but our sensitivity analyses suggest that these issues will not alter the bottom line.
Numerous studies have shown that it is expensive to $c$ alter practice patterns. These investments will necessarily be made by organizations that need to ask hard questions about the tradeoffs between costs and consequences of $O$ using their resources to promote change in the clinical community. It behooves the National Institutes of Health to make these economic tradeoffs explicit when they create guidelines for clinical practice. This process $\frac{T}{0}$ does not necessitate making recommendations based on ? economic outcomes. The economic section of JNC VI $\overrightarrow{\vec{B}}$ does not provide explicit quantitative data and thus is of $\frac{\mathscr{\infty}}{0}$ little use for decision makers. In the case of managing hypertension, we show that following the JNC VI rec- $\frac{\bar{\sigma}}{D}$ ommendations is economically and clinically a win-win $\mathbb{Q}$ situation. We expect other cases to be less clear.

In an era when economic factors can and often do influence medical decisions, we believe it is important to $\dot{\omega}$ have high-quality, objective economic data available $\vec{N}$ alongside clinical data for common conditions such as $\frac{N}{0}$ hypertension. The National High Blood Pressure Edu- 을 cation Program is an ideal and yet unrealized forum for? such information.

Scott D. Ramsey, MD, PhD is Center for Cost and Outcomes Research $\stackrel{\vec{N}}{-}$ University of Washington 은 Sean D. Sullivan, $\mathrm{PhD} \rightarrow$ Department of Pharmacy $\mathbb{D}$ University of Washington $\frac{\mathbb{D}}{\mathbb{D}}$ Seattle, Wash

\section{References}

1. Siegel D, Lopez J. Trends in antihypertensive drug use in the United States: do the JNC V recommendations affect prescribing? Fifth Joint National Commission on the Detection, Evaluation, and Treatment of High Blood Pressure. JAMA 1997;278:1745-8.

2. Lenfant $\mathrm{C}$. JNC guidelines: is the message getting through? Joint National Commission on Detection, Evaluation, and Treatment of High Blood Pressure. JAMA 1997;278: 1778-9.

\section{In-Flight Radiation}

To the Editor: Your readers should be aware that the article by Robert F. Barish on in-flight radiation ${ }^{1}$ is based $\frac{0}{0}$ on a now-discredited linear no-threshold hypothesis of $\mathrm{O}$ radiation health risk. Nuclear Issues ${ }^{2}$ reports that the $\vec{\sim}$ American Nuclear Society has issued a position state- o ment to the effect that "there is insufficient scientific evidence to support use of the linear no threshold hypothesis (LNTH) in the projection of the health effects $N$ of low-level radiation on which regulation of low levels of $\frac{\omega}{\sigma}$ radiation adopted by international and national radiation protection authorities is based."

Also, the US National Council on Radiation Protec- $\stackrel{\infty}{?}$ tion (NCRP) ${ }^{3}$ has stated that "few experimental studies, and essentially no human data, can be said to prove or $\frac{O}{\mathbb{D}}$ even to provide direct support for the concept of collec- $\frac{O}{8}$ tive dose with its implicit uncertainties of no-threshold $\cong$ 